\title{
Review of: "Ehlers-Danlos Syndrome"
}

\author{
Dacre Knight ${ }^{1}$ \\ 1 Mayo Foundation for Medical Education and Research
}

Potential competing interests: The author(s) declared that no potential competing interests exist.

Would include "tissue fragility" as described fragility is not limited to skin involvement. 